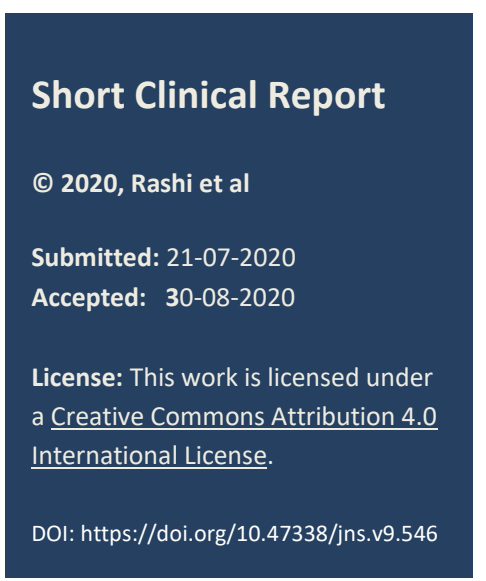

\section{lleocecal web with central perforation in a neonate: A peculiar inclusion to the scarce literature of ileocecal valve anomaly}

Rashi Rashi, Mohd. Mokarram Ali,* Amit Kumar, Amit Kumar Sinha, Bindey Kumar

Department of Pediatric Surgery, All India Institute of Medical Sciences Patna

Correspondence*: Mohd. Mokarram Ali, Department of Pediatric Surgery, All India Institute of Medical Sciences, Patna E-mail: mohdmokarramali1990@gmail.com

\section{CASE PRESENTATION}

A 12-day-old male neonate presented in the emergency room with constipation for three days and repeated episodes of non-bilious vomiting for one day. The patient had a history of delayed passage of meconium (after 48 hours) at birth. On examination, the abdomen was distended but soft, and bowel sounds present. X-ray abdomen showed multiple air-fluid levels. Suspecting Hirschsprung's disease, saline enema was started. The patient started passing the stool and the abdominal distension settled down, thus feeding was resumed. The patient tolerated the feeds and started passing stool without enema. The patient was discharged and followed-up in the outpatient department (OPD). After two weeks, the patient again presented with abdominal distension and constipation. Rectal washout was given but the patient passed small amount of stool and abdominal distension persisted. Contrast enema performed showed narrow lumen of the entire colon, and the contrast could not pass proximal to the ileocecal junction (Fig. 1A).

On exploration, hugely distended small bowel loops were found with collapsed colon. We suspected obstruction at the level of ileocecal junction. Thickening of the ileal wall proximal to obstruction site was also noted. A longitudinal enterotomy performed at ileocecal junction revealed a mucosal web at the ileocecal junction with central perforation (Fig.1B). The web was excised and enterotomy closed transversely. Proximal diversion as ileostomy was given. Immediate postoperative recovery was uneventful, but the patient developed signs of sepsis on 5th postoperative day (sluggish movement of ileostomy, abdominal distention, thrombocytopenia, hypoglycemia, and seizures). Antibiotics upgraded, hypoglycemia corrected, and the patient was put on mechanical ventilation. The patient was weaned off the mechanical ventilation on improvement and discharged after establishing full oral feeding. On follow-up the patient is thriving well; and ileostomy reversal has been planned.

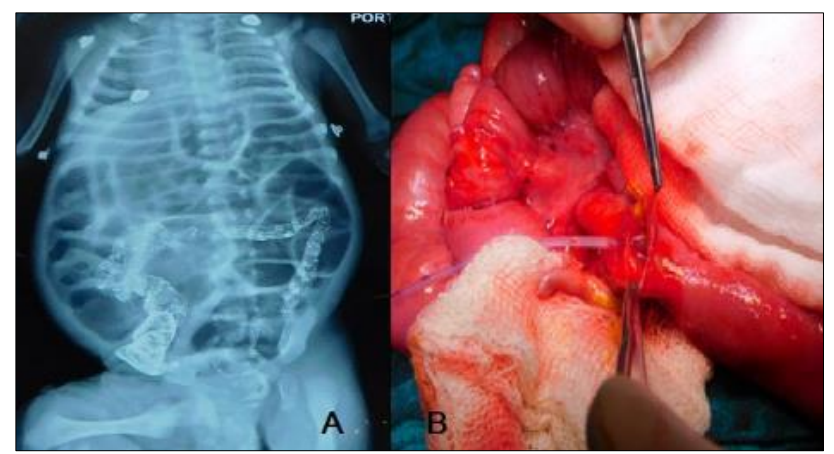

Figure 1: A) Contrast enema showing small caliber colon with non-passage of the contrast beyond the ileocecal region and grossly dilated proximal bowel loops. B) Enterotomy showing web at the ileocecal junction with a central perforation.

\section{DISCUSSION}

Intestinal atresia of the ileocecal junction is exceedingly rare. Presence or absence of ileocecal valve affects the surgical management and prognosis in such cases especially with short bowel.[1,2] Isolated ileocecal valve atresia is a rare entity with only 10 cases have been reported in the literature.[2,3-7] Mousavi et al. reported a case of type-I ileocecal valve atresia consisted of a mucosal diaphragm. [8] In the index case, a mucosal web with a central perforation was obstructing the ileocecal valve. Central perforation of the mucosal web in the index case led to a delayed presentation as small amount of the stool kept on pouring into the large bowel. This was also the reason of labelling it a case of Hirschsprung disease preoperatively.

Different surgical techniques have been suggested for the ileocecal valve atresia depending on the intraoperative findings. Resection of the atretic segment, followed by an ileocolonic anastomosis is the most frequently employed approach.[1,2,4,7] In our case, there 
was only a mucosal web with central perforation without any atretic segment. Therefore, we decided to excise the web and preserve the ileocecal segment. A proximal diverting ileostomy was deemed necessary due to poor health status of the baby and fearing anastomotic leakage owing to its proximity with ileocecal valve.

To summarize, this report described a mucosal web with central perforation in the ileocecal valve region obscuring the identification of valve itself. Management of such cases depend on intraoperative findings and clinical status of the patient.

\section{REFERENCES}

1. Cserni T, Magyar A, Németh T, Paran TS, Csízy I, Józsa T. Atresia of the ileocecal junction with agenesis of the ileocecal valve and vermiform appendix: report of a case. Surg Today. 2006; 36:1126-8.

2. Srivastava P, Gangopadhyay AN, Gupta DK, Sharma SP, Upadhyaya VD, Kumar V, et al. Atresia of ileocecal junction, ileocecal valve: Rare variants of bowel atresia. J Indian Assoc Pediatr Surg. 2009; 14:117-8.

3. Cacciari A, Mordenti M, Ceccarelli PL, Ruggeri G. Ileocaecal valve atresia: our surgical approach. Eur J Pediatr Surg. 2004; 14:435-9.

4. Kassira N, Keisling M, Prasad R. True atresia of the ileocecal valve: Diagnosis and management of an
Acknowledgements: Nil

Conflict of Interest: None declared

Source of Support: Nil

Consent to Publication: Author(s) declared taking informed written consent for the publication of clinical photographs/material (if any used), from the legal guardian of the patient with an understanding that every effort will be made to conceal the identity of the patient, however it cannot be guaranteed.

Author Contributions: Author(s) declared to fulfil authorship criteria as devised by ICMJE and approved the final version.

extremely rare entity. $J$ Pediatr Surg Case Rep. [Internet]. 2015 [cited 2020 Aug 14]; 3:117-9. Available from: https://doi.org/10.1016/j.epsc.2015.01.010.

5. Grassi R, Pinto A, Valente T, Rossi G, Catalano O, Rotondo A, et al. Massive enterolithiasis associated with ileal dysgenesis. Br J Radiol. 1997; 70:207-9.

6. AlZaiem M, Al-Garni AF, Almaghrebi A, Zaiem F. Isolated ileocecal valve atresia. Ann Pediatr Surg. 2018; 14:211-3.

7. Ein SH, Venugopal S, Mancer K. Ileocaecal atresia. J Pediatr Surg. 1985; 20:525-8.

8. Mousavi SA, Sarparast L. Ileocecal valve atresia: Introduction of a new surgical approach. Afr J Paediatr Surg. 2014; 11:77. 